\title{
Total Synthesis of Brasoside and Littoralisone
}

\author{
Ian K. Mangion and David W. C. MacMillan* \\ Division of Chemistry and Chemical Engineering, California Institute of \\ Technology, Pasadena, CA 91125 \\ Supporting Information
}

General Information. Commercial reagents were purified prior to use following the guidelines of Perrin and Armarego. ${ }^{1}$ All solvents were purified according to the method of Grubbs. ${ }^{2} \mathrm{CH}_{3} \mathrm{CN}$ was stored under argon over activated molecular sieves. TMSOTf was doubly distilled from $\mathrm{CaH}_{2}$ prior to use. Non-aqueous reagents were transferred under argon via syringe or cannula. Organic solutions were concentrated under reduced pressure on a Büchi rotary evaporator using a water bath. Chromatographic purification of products was accomplished using forced-flow chromatography on ICN $6032-64$ mesh silica gel or Iatrobeads ${ }^{\square}$ according to the method of Still. ${ }^{3}$ Thin-layer chromatography (TLC) was performed on EM Reagents 0.25 $\mathrm{mm}$ silica gel $60-\mathrm{F}$ plates. Visualization of the developed chromatogram was performed by fluorescence quenching or by anisaldehyde stain.

${ }^{1} \mathrm{H}$ and ${ }^{13} \mathrm{C}$ NMR spectra were recorded on a Varian Mercury $300(300 \mathrm{MHz}$ and 75 $\mathrm{MHz})$ or 500 (500 MHz and $125 \mathrm{MHz}$ ) Spectrometer as noted, and are internally referenced to residual protio solvent signals. Data for ${ }^{1} \mathrm{H}$ NMR are reported as follows: chemical shift $(\square$ $\mathrm{ppm})$, multiplicity $(\mathrm{s}=$ singlet, $\mathrm{d}=$ doublet, $\mathrm{t}=$ triplet, $\mathrm{q}=$ quartet, $\mathrm{m}=$ multiplet $)$, integration, coupling constant $(\mathrm{Hz})$ and assignment. Where appropriate, the notations $\mathrm{H} 1, \mathrm{H} 2, \mathrm{H} 3, \mathrm{H} 4, \mathrm{H} 5$, and $\mathrm{H} 6$ have been used to refer to protons residing on the denoted carbons in a sugar. Data for ${ }^{13} \mathrm{C}$ NMR are reported in terms of chemical shift. IR spectra were recorded on a Perkin Elmer Paragon 1000 spectrometer and are reported in terms of frequency of absorption $\left(\mathrm{cm}^{-1}\right)$. Mass spectra were obtained from the California Institute of Technology Mass Spectral Facility. Optical rotations were measured on a Jasco P-1010 polarimeter, and $[\square]_{D}$ values are reported in $10^{-1} \mathrm{dg} \mathrm{cm}^{2} \mathrm{~g}^{-1}$. 
(S)-3,7-dimethyloct-6-enyl 2,4,6-trimethylbenzoate. To a stirring solution of (-)citronellol ( $8.8 \mathrm{~mL}, 48 \mathrm{mmol})$, pyridine $(7.8 \mathrm{~mL}, 96 \mathrm{mmol})$, and DMAP (100 mg, $0.82 \mathrm{mmol})$ in $\mathrm{CH}_{2} \mathrm{Cl}_{2}(250 \mathrm{~mL})$ was added 2,4,6-trimethylbenzoyl chloride $(9.0 \mathrm{~mL}, 53 \mathrm{mmol})$. After $10 \mathrm{~h}$ the solution was diluted in $500 \mathrm{~mL} \mathrm{Et}_{2} \mathrm{O}$ and washed with $150 \mathrm{~mL}$ saturated solutions of $\mathrm{NH}_{4} \mathrm{Cl}$, $\mathrm{NaHCO}_{3}$, and $\mathrm{NaCl}$. The organic layer was dried over $\mathrm{Na}_{2} \mathrm{SO}_{4}$ and concentrated in vacuo. Flash chromatography (95:5 pentane: $\mathrm{Et}_{2} \mathrm{O}$ ) afforded the title compound as a clear, colorless oil in $99 \%$ yield (14.4 g, 47.5 mmol). IR (film) 2956, 2921, 2852, 1778, 1726, 1613, 1453, 1436, 1376, 1264, 1214, 1170, $1083 \mathrm{~cm}^{-1}$; ${ }^{1} \mathrm{H}$ NMR (300 MHz, $\left.\mathrm{CDCl}_{3}\right) \square 6.85$ (s, 2H, Ar-H); 5.09 (m, 1H, $\left.\left(\mathrm{CH}_{3}\right)_{2} \mathrm{C}=\mathrm{CH}\right) ; 4.35$ (m, 2H, CH $\left.\mathbf{H}_{2} \mathrm{OMes}\right) ; 2.29$ (s, 9H, $\left.\operatorname{ArCH}_{3}\right) ; 1.96\left(\mathrm{~m}, 2 \mathrm{H}, \mathrm{C}=\mathrm{CHCH}_{2}\right) ; 1.82-$ $1.26\left(\mathrm{~m}, 11 \mathrm{H}, \mathrm{C}=\mathrm{C}\left(\mathrm{CH}_{3}\right)_{2}, \mathrm{CH}_{2} \mathrm{CHCH}_{3}, \mathrm{CHCH}_{3}\right) ; 1.02\left(\mathrm{~d}, 3 \mathrm{H}, J=6.6 \mathrm{~Hz}, \mathrm{CHCH}_{3}\right) ;{ }^{13} \mathrm{C} \mathrm{NMR}$ $\left(75 \mathrm{MHz}, \mathrm{CDCl}_{3}\right) \square 170.5,151.1,139.3,135.2,131.6,131.4,128.9,128.6,124.9,63.5,40.6$, $37.2,35.9,29.7,25.9,25.7,21.2,20.0,19.5,17.8$; HRMS (FAB+) exact mass calculated for [M $+\mathrm{H}]^{+}\left(\mathrm{C}_{20} \mathrm{H}_{31} \mathrm{O}_{2}\right)$ requires $m / z$ 303.2324, found $m / z$ 303.2333. $[\square]_{D}^{25}=-1.84\left(\mathrm{c}=1.0, \mathrm{CHCl}_{3}\right)$.

(S)-5-formyl-3-methylpentyl 2,4,6-trimethylbenzoate (7). A solution of (S)-3,7dimethyloct-6-enyl 2,4,6-trimethylbenzoate $(9.0 \mathrm{~g}, 30 \mathrm{mmol})$ and pyridine $(2.6 \mathrm{~mL}, 45 \mathrm{mmol})$ in $\mathrm{CH}_{2} \mathrm{Cl}_{2} / \mathrm{MeOH}(140 \mathrm{~mL} / 15 \mathrm{~mL})$ was cooled to $-78^{\circ} \mathrm{C}$. Ozone was bubbled through the solution until a dark blue color developed. At this time triphenylphosphine $(8.6 \mathrm{~g}, 33 \mathrm{mmol})$ was added and the resulting mixture was stirred for $3 \mathrm{~h}$ allowing it to reach $0{ }^{\circ} \mathrm{C}$. After concentration, flash chromatography (19:1-10:1 pentane: $\mathrm{Et}_{2} \mathrm{O}$ ) afforded the title compound as a clear, colorless oil in 96\% yield (7.96 g, 28.8 mmol). IR (film) 2959, 2926, 2873, 1724, 1612, 1458, 1435, 1380, 1266, 1170, 1085, 958.7, $853.1 \mathrm{~cm}^{-1}$; ${ }^{1} \mathrm{H}$ NMR (300 MHz, $\left.\mathrm{CDCl}_{3}\right) \square 9.77$ (t, $\left.1 \mathrm{H}, J=1.8 \mathrm{~Hz}, \mathrm{CHO}\right)$; $\square$ 6.85 (s, 2H, Ar-H); 4.36 (m, 2H, CH $\mathbf{H}_{2} \mathrm{OMes}$ ); 2.46 (m, 2H, $\mathbf{C H}_{2} \mathrm{CHO}$ ); 2.28 (s, 9H, $\mathrm{ArCH}_{3}$ ); 1.83-1.46 (m, 5H, $\left.\mathrm{CH}_{2} \mathrm{CHCH}_{3}, \mathrm{CHCH}_{3}\right) ; 0.96\left(\mathrm{~d}, 3 \mathrm{H}, J=6.3 \mathrm{~Hz}, \mathrm{CHCH}_{3}\right) ;{ }^{13} \mathrm{C}$ NMR $(75 \mathrm{MHz}$, $\left.\mathrm{CDCl}_{3}\right) \square 202.4,170.3,151.1,139.4,135.1,131.4,128.6,128.5,63.1,41.6,35.6,29.6,28.9$, 21.3, 20.0, 19.2, 19.1; HRMS (EI+) exact mass calculated for $[\mathrm{M}]^{+}\left(\mathrm{C}_{17} \mathrm{H}_{24} \mathrm{O}_{3}\right)$ requires $\mathrm{m} / \mathrm{z}$ 276.1728, found $m / z$ 276.1726. $[\square]_{D}^{25}=-0.33\left(\mathrm{c}=1.0, \mathrm{CHCl}_{3}\right)$.

$(E, 3 R, 5 S)$-7-(methoxycarbonyl)-5-hydroxy-3-methylhept-6-enyl 2,4,6-trimethylbenzoate (8). D-Proline (530 mg, $4.6 \mathrm{mmol})$ was added to a stirring solution of 7 (3.12 g, 11.3 mmol) and nitrosobenzene $(1.21 \mathrm{~g}, 11.3 \mathrm{mmol})$ in DMSO $(45 \mathrm{~mL})$. After $0.5 \mathrm{~h}$ the solution 
became a bright orange, at which time it was cooled to $-15{ }^{\circ} \mathrm{C}$. A premixed solution of methyl diethyl phosphonoacetate $(6.0 \mathrm{~mL}, 34 \mathrm{mmol})$, 1,8-diazabicyclo[5.4.0]undec-7-ene (5.1 mL, 34 mmol) and lithium chloride (1.44 g, $34 \mathrm{mmol})$ in $\mathrm{CH}_{3} \mathrm{CN}(45 \mathrm{~mL})$ was added over 5 min via cannula. After 15 min the solution was diluted with $\mathrm{MeOH}(150 \mathrm{~mL})$ and $\mathrm{NH}_{4} \mathrm{Cl}(1.8 \mathrm{~g}, 34$ mmol) was added. The resulting mixture was allowed to warm to room temperature and stand for $2 \mathrm{~d}$. At this time the solution was diluted with $\mathrm{Et}_{2} \mathrm{O}(700 \mathrm{~mL})$, and washed successively with $200 \mathrm{~mL}$ saturated solutions of $\mathrm{NH}_{4} \mathrm{Cl}, \mathrm{NaHCO}_{3}$, and $\mathrm{NaCl}$. The aqueous layers were extracted with $3 \times 100 \mathrm{~mL} \mathrm{CH}_{2} \mathrm{Cl}_{2}$, and the combined organic layers were dried over $\mathrm{Na}_{2} \mathrm{SO}_{4}$ and concentrated in vacuo. Flash chromatography (5:1-3:2 pentane: $\mathrm{Et}_{2} \mathrm{O}$ ) afforded the title compound as a clear, colorless oil in 56\% yield ( $2.2 \mathrm{~g}, 6.33 \mathrm{mmol})$. IR (film) 3479, 2958, 2925, 1723, 1612, 1455, 1436, 1267, 1170, 1085, 1036, 983.8, $853.0 \mathrm{~cm}^{-1} ;{ }^{1} \mathrm{H}$ NMR (300 MHz, $\left.\mathrm{CDCl}_{3}\right)$ $\square 6.95$ (dd, 1H, $J=15.6,4.8 \mathrm{~Hz}, \mathrm{C}=\mathrm{CHCHOH}) ; 6.85$ (s, 2H, Ar-H); 6.05 (dd, 1H, $J=15.6,1.6$ $\mathrm{Hz}, \mathrm{C}=\mathrm{CHCO}_{2} \mathrm{Me}$ ); 4.45-4.27 (m, 3H, $\left.\mathbf{C H}_{2} \mathrm{OMes}, \mathbf{C H O H}\right) ; 3.74$ (s, 3H, OCH $\mathbf{H}_{3}$; 2.28 (s, 9H, $\left.\mathrm{ArCH}_{3}\right) ; 1.98-1.24\left(\mathrm{~m}, 5 \mathrm{H}, \mathrm{CH}_{2} \mathrm{CHCH}_{3}, \mathrm{CHCH}_{3}\right) ; 1.01\left(\mathrm{~d}, 3 \mathrm{H}, J=6.6 \mathrm{~Hz}, \mathrm{CHCH}_{3}\right) ;{ }^{13} \mathrm{C} \mathrm{NMR}$ $\left(75 \mathrm{MHz}, \mathrm{CDCl}_{3}\right) \square$ 170.6, 167.3, 151.8, 139.4, 135.2, 135.0, 131.4, 128.6, 128.5, 119.4, 68.6, 63.2, 51.7, 43.8, 36.3, 26.6, 21.3, 20.0, 19.9 19.1; HRMS (EI+) exact mass calculated for [M + $\mathrm{H}]^{+}\left(\mathrm{C}_{20} \mathrm{H}_{29} \mathrm{O}_{5}\right)$ requires $m / z 349.2015$, found $m / z 349.2023 .[\square]_{D}^{25}=-5.78\left(\mathrm{c}=1.0, \mathrm{CHCl}_{3}\right)$.

\section{(E,3R,5S)-7-(methoxycarbonyl)-5-(tert-butyl-diphenyl-silanyloxy)-3-methylhept-6-}

enyl 2,4,6-trimethylbenzoate. tert-Butylchlorodiphenylsilane $(4.6 \mathrm{~mL}, 17.8 \mathrm{mmol})$ was added to a stirring solution of $8(3.1 \mathrm{~g}, 8.9 \mathrm{mmol})$, imidazole (1.5 g, $22.2 \mathrm{mmol})$, and DMAP (100 $\mathrm{mg}$, $0.82 \mathrm{mmol})$ in DMF $(20 \mathrm{~mL})$. After $12 \mathrm{~h}$ the solution was diluted in $250 \mathrm{~mL} \mathrm{Et}_{2} \mathrm{O}$ and washed with $50 \mathrm{~mL}$ saturated solutions of $\mathrm{NH}_{4} \mathrm{Cl}, \mathrm{NaHCO}_{3}$, and $\mathrm{NaCl}$. The organic layer was dried over $\mathrm{Na}_{2} \mathrm{SO}_{4}$ and concentrated in vacuo. Flash chromatography (85:15 pentane:Et $\mathrm{Et}_{2} \mathrm{O}$ ) afforded the title compound as a clear, colorless oil in 97\% yield (5.1 g, $8.63 \mathrm{mmol})$. IR (film) 3072, 3049, 2957, 2931, 2858, 1726, 1612, 1472, 1428, 1362, 1267, 1170, 1112, 1085, 1036, 852.6, 821.8, 740.9, 702.0, 607.9, 504.3 $\mathrm{cm}^{-1} ;{ }^{1} \mathrm{H}$ NMR (300 MHz, $\mathrm{CDCl}_{3}$ ) $\square 7.81-7.34$ (m, 10H, SiPhH); 6.90 (dd, $1 \mathrm{H}, J=15.6,5.4 \mathrm{~Hz}, \mathrm{C}=\mathrm{CHCHOSi}) ; 6.87(\mathrm{~s}, 2 \mathrm{H}, \operatorname{Ar}-\mathbf{H}) ; 5.86(\mathrm{~d}, 1 \mathrm{H}, J=15.6 \mathrm{~Hz}$, $\left.\mathrm{C}=\mathrm{CHCO}_{2} \mathrm{Me}\right) ; 4.39(\mathrm{~m}, 1 \mathrm{H}, \mathrm{CHOSi}) ; 4.20\left(\mathrm{~m}, 2 \mathrm{H}, \mathrm{CH}_{2} \mathrm{OMes}\right) ; 3.72\left(\mathrm{~s}, 3 \mathrm{H}, \mathrm{OCH}_{3}\right) ; 2.29(\mathrm{~s}$, 9H, $\left.\operatorname{ArCH}_{3}\right) ; 1.76-1.28$ (m, 5H, $\left.\mathbf{C H}_{2} \mathrm{CHCH}_{3}, \mathrm{CHCH}_{3}\right) ; 1.10$ (s, 9H, $\left.\mathrm{SiC}\left(\mathrm{CH}_{3}\right)_{3}\right) ; 0.90$ (d, 3H, $J=$ $\left.6.6 \mathrm{~Hz}, \mathrm{CHCH}_{3}\right) ;{ }^{13} \mathrm{C} \mathrm{NMR}\left(75 \mathrm{MHz}, \mathrm{CDCl}_{3}\right) \square 170.5,167.1,150.6,139.4,136.2,136.1,136.0$, 
135.6, 135.5, 135.3, 135.1, 134.0, 130.5, 130.3, 130.1, 129.9, 128.6, 128.1, 128.0, 127.9, 127.8, 120.1, 71.3, 63.2, 51.8, 45.1, 35.8, 27.3, 26.8, 26.7, 26.4, 21.4, 20.0, 19.9, 19.6; HRMS (FAB+) exact mass calculated for $[\mathrm{M}+\mathrm{H}]^{+}\left(\mathrm{C}_{36} \mathrm{H}_{47} \mathrm{O}_{5} \mathrm{Si}\right)$ requires $\mathrm{m} / \mathrm{z}$ 587.3193, found $\mathrm{m} / \mathrm{z}$ 587.3192. $[\square]_{D}^{25}=-12.13\left(\mathrm{c}=1.0, \mathrm{CHCl}_{3}\right)$.

\section{$(E, 4 S, 6 R)$-6-methyl-4-(tert-butyl-diphenyl-silanyloxy)-oct-2-ene-1,8-diol. $\quad$ A $1 \mathrm{M}$} solution of diisobutylaluminum hydride in hexanes $(75 \mathrm{~mL}, 75 \mathrm{mmol})$ was slowly added to a stirred $-78{ }^{\circ} \mathrm{C}$ solution of (E,3R,5S)-7-(methoxycarbonyl)-5-(tert-butyl-diphenyl-silanyloxy)-3methylhept-6-enyl 2,4,6-trimethylbenzoate $(7.4 \mathrm{~g}, 12.6 \mathrm{mmol})$ in $\mathrm{Et}_{2} \mathrm{O}(250 \mathrm{~mL})$. After $30 \mathrm{~min}$ $\mathrm{MeOH}\left(3 \mathrm{~mL}\right.$ ) was slowly added, followed by dilution with $250 \mathrm{~mL} \mathrm{Et}_{2} \mathrm{O}$ and warming to room temperature. Saturated Rochelle's salt $(300 \mathrm{~mL})$ was then added, followed by vigorous stirring overnight. The aqueous layer was then separated and extracted with $2 \times 100 \mathrm{~mL} \mathrm{CH}_{2} \mathrm{Cl}_{2}$ and $\mathrm{Et}_{2} \mathrm{O}$. The combined organic layers were dried over $\mathrm{Na}_{2} \mathrm{SO}_{4}$ and concentrated in vacuo. Flash chromatography (1:3 pentane: $\mathrm{Et}_{2} \mathrm{O}$ ) afforded the title compound as a clear, colorless oil in $96 \%$ yield (5.0 g, $12.1 \mathrm{mmol}$ ). IR (film) 3338, 3072, 3049, 2956, 2930, 2858, 1472, 1462, 1428, 1362, 1112, 1057, 972.6, 822.2, 739.1, 702.2, 612.4, $504.8 \mathrm{~cm}^{-1} ;{ }^{1} \mathrm{H}$ NMR (300 MHz, $\left.\mathrm{CDCl}_{3}\right) \square 7.70-$ $7.32(\mathrm{~m}, 10 \mathrm{H}, \mathrm{SiPhH}) ; 5.56$ (ddt, $1 \mathrm{H}, J=16.2,7.5,1.2 \mathrm{~Hz}, \mathrm{C}=\mathrm{CHCH}_{2} \mathrm{OH}$ ); 5.38 (ddt, $1 \mathrm{H}, J=$ $16.2,5.5,0.6 \mathrm{~Hz}, \mathrm{C}=\mathrm{CHCHOSi}) ; 4.24(\mathrm{~m}, 1 \mathrm{H}, \mathrm{CHOSi}) ; 3.87\left(\mathrm{~d}, 2 \mathrm{H}, J=5.5 \mathrm{~Hz}, \mathrm{CHCH}_{2} \mathrm{OH}\right)$; $3.56\left(\mathrm{~m}, 2 \mathrm{H}, \mathrm{CH}_{2} \mathrm{CH}_{2} \mathrm{OH}\right) ; 1.76-1.18\left(\mathrm{~m}, 5 \mathrm{H}, \mathrm{CH}_{2} \mathrm{CHCH}_{3}, \mathrm{CHCH}_{3}\right) ; 1.06$ (s, 9H, $\left.\mathrm{SiC}\left(\mathrm{CH}_{3}\right)_{3}\right)$; $0.78\left(\mathrm{~d}, 3 \mathrm{H}, J=6.9 \mathrm{~Hz}, \mathrm{CHCH}_{3}\right) ;{ }^{13} \mathrm{C} \mathrm{NMR}\left(75 \mathrm{MHz}, \mathrm{CDCl}_{3}\right) \square 136.4,136.2,135.0,134.8$, $129.9,129.8,129.6,129.5,128.0,127.9,127.8,127.6,72.5,63.2,61.0,45.6,39.9,27.4,27.3$, 27.2, 25.7, 20.3, 19.6; HRMS (FAB+) exact mass calculated for $[\mathrm{M}+\mathrm{H}]^{+}\left(\mathrm{C}_{25} \mathrm{H}_{37} \mathrm{O}_{3} \mathrm{Si}\right)$ requires $m / z$ 413.2512, found $m / z$ 413.2513. $[\square]_{D}^{25}=-19.57\left(\mathrm{c}=1.0, \mathrm{CHCl}_{3}\right)$.

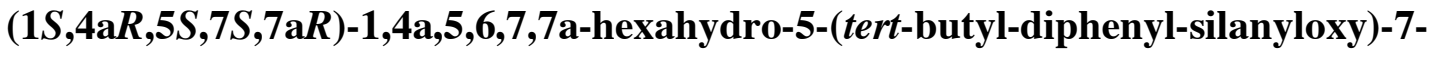

methylcyclopenta[c]pyran-1-yl acetate (11). Dess-Martin periodinane $(2.54 \mathrm{~g}, 6.1 \mathrm{mmol})$ was added to a stirred solution of ( $E, 4 S, 6 R)$-6-methyl-4-(tert-butyl-diphenyl-silanyloxy)-oct-2-ene1,8-diol (1.07 g, $2.60 \mathrm{mmol})$ in $\mathrm{CH}_{2} \mathrm{Cl}_{2}(26 \mathrm{~mL})$. After $40 \mathrm{~min}$ the reaction was concentrated and extracted with $3 \times 50 \mathrm{~mL}$ pentane. The combined organics were concentrated in vacuo, providing $1.0 \mathrm{~g}$ (94\% yield, $2.44 \mathrm{mmol})$ of the corresponding dialdehyde, which was immediately redissolved in DMSO (61 $\mathrm{mL})$. L-Proline $(93 \mathrm{mg}, 0.80 \mathrm{mmol})$ was added to this stirred solution 
in one portion. After $5 \mathrm{~h}$, the reaction was warmed to $40{ }^{\circ} \mathrm{C}$ and stirred at this temperature for 60 $\mathrm{h}$ at which point TLC analysis showed completion. The reaction was then cooled to $0{ }^{\circ} \mathrm{C}$, and acetic anhydride $(2.3 \mathrm{~mL}, 24 \mathrm{mmol})$ was added, followed by pyridine $(1.0 \mathrm{~mL}, 12 \mathrm{mmol})$ and DMAP $\left(25 \mathrm{mg}, 0.23 \mathrm{mmol}\right.$ ). After $15 \mathrm{~min}$ the reaction was diluted with $200 \mathrm{~mL} \mathrm{Et}_{2} \mathrm{O}$ and washed with $50 \mathrm{~mL}$ saturated solutions of $\mathrm{NH}_{4} \mathrm{Cl}, \mathrm{NaHCO}_{3}$, and $\mathrm{NaCl}$. The aqueous layers were then extracted with $2 \times 50 \mathrm{~mL} \mathrm{CH}_{2} \mathrm{Cl}_{2}$ and $2 \times 50 \mathrm{~mL} \mathrm{Et} \mathrm{Et}_{2} \mathrm{O}$. The combined organic layers were dried over $\mathrm{Na}_{2} \mathrm{SO}_{4}$ and concentrated in vacuo. Flash chromatography (19:1 pentane: $\mathrm{Et}_{2} \mathrm{O}$ ) afforded the title compound as a clear, colorless oil in $83 \%$ yield (910 mg, $2.02 \mathrm{mmol}$ ). IR (film) 3072, 2956, 2931, 2858, 1761, 1652, 1472, 1428, 1362, 1211, 1112, 1026, 953.8, $702.3 \mathrm{~cm}^{-1} ;{ }^{1} \mathrm{H}$ NMR $\left(300 \mathrm{MHz}, \mathrm{CDCl}_{3}\right) \square 7.70-7.35(\mathrm{~m}, 10 \mathrm{H}, \mathrm{SiPhH}) ; 6.35(\mathrm{dd}, 1 \mathrm{H}, J=6.3,2.4 \mathrm{~Hz}$, $\mathrm{OCH}=\mathrm{CH}) ; 6.08(\mathrm{~d}, 1 \mathrm{H}, J=6.6, \mathrm{CHOAc}) ; 5.05(\mathrm{dd}, 1 \mathrm{H}, J=6.3,2.7 \mathrm{~Hz}, \mathrm{OCH}=\mathrm{CH}) ; 4.32(\mathrm{dd}$, $1 \mathrm{H}, J=12.0,6.6 \mathrm{~Hz}, \mathrm{CHOSi}) ; 2.59(\mathrm{~m}, 1 \mathrm{H}, \mathrm{C}=\mathrm{CHCH}) ; 2.10$ (s, 3H, OC(O)CH $)$; 2.08-1.84 (m, $\left.3 \mathrm{H}, \mathrm{CH}_{2} \mathrm{CHCH}_{3}, \mathrm{CHCH}_{3}, \mathbf{C H C H O A c}\right) ; 1.24\left(\mathrm{~m}, 1 \mathrm{H}, \mathrm{CH}_{2} \mathrm{CHCH}_{3}\right) ; 1.08$ (s, 9H, $\left.\mathrm{SiC}\left(\mathrm{CH}_{3}\right)_{3}\right) ; 0.94$ $\left(\mathrm{d}, 3 \mathrm{H}, J=6.9 \mathrm{~Hz}, \mathrm{CHCH}_{3}\right) ;{ }^{13} \mathrm{C} \mathrm{NMR}\left(75 \mathrm{MHz}, \mathrm{CDCl}_{3}\right) \square$ 170.0, 141.0, 136.1, 136.0, 135.9, 135.8, 130.0, 129.9, 129.8, 127.9, 127.8, 127.7, 101.3, 91.6, 75.3, 46.0, 40.9, 38.8, 31.6, 27.2, 21.5, 21.4, 19.5; HRMS (FAB+) exact mass calculated for $[\mathrm{M}+\mathrm{H}]^{+}\left(\mathrm{C}_{27} \mathrm{H}_{35} \mathrm{O}_{4} \mathrm{Si}\right)$ requires $\mathrm{m} / \mathrm{z}$ 451.2305, found $m / z$ 451.2305. $[\square]_{D}^{25}=-104.1\left(\mathrm{c}=1.0, \mathrm{CHCl}_{3}\right)$.

\section{$(1 S, 4 a S, 5 S, 7 S, 7 a R)$-4-formyl-1,4a,5,6,7,7a-hexahydro-5-(tert-butyl-diphenyl-} silanyloxy)-7-methylcyclopenta[c]pyran-1-yl acetate. DMF $(6 \mathrm{~mL})$ that had been stored over activated molecular sieves for at least $24 \mathrm{~h}$ was added to a flame-dried schlenk flask and cooled to $-20^{\circ} \mathrm{C}$. Freshly distilled phosphorous oxychloride $(0.84 \mathrm{~mL}, 9.0 \mathrm{mmol})$ was added dropwise with stirring. The mixture was allowed to slowly warm to room temperature over the course of 1 $\mathrm{h}$, and then stirred at that temperature for an additional $1 \mathrm{~h}$. A solution of $11(580 \mathrm{mg}, 1.3 \mathrm{mmol})$ in DMF ( $3 \mathrm{~mL}$ ) was then added dropwise, and the resulting mixture was warmed to $40{ }^{\circ} \mathrm{C}$. After $60 \mathrm{~h}$ the reaction was cooled to $-20^{\circ} \mathrm{C}$, quenched by addition of $10 \mathrm{~mL}$ of a saturated solution of $\mathrm{NaHCO}_{3}$, and extracted with $3 \times 50 \mathrm{~mL} \mathrm{Et} 2 \mathrm{O}$. The combined organics were dried over $\mathrm{Na}_{2} \mathrm{SO}_{4}$ and concentrated in vacuo. Flash chromatography (5:1 pentane: $\left.\mathrm{Et}_{2} \mathrm{O}\right)$ afforded the title compound as a clear, colorless oil in 73\% yield (451 mg, $0.94 \mathrm{mmol}$ ). IR (film) 3072, 2957, 2931, 2858, 1766, 1677, 1633, 1472, 1428, 1367, 1215, 1183, 1091, 1071, 821.6, 740.0, 704.5 $\mathrm{cm}^{-1}$; ${ }^{1} \mathrm{H}$ NMR (300 MHz, $\left.\mathrm{CDCl}_{3}\right) \square 9.18(\mathrm{~s}, 1 \mathrm{H}, \mathrm{CHO}) ; 7.67-7.29$ (m, 11H, SiPhH, OCH=C); 
6.45 (d, 1H, $J=9.3$, CHOAc); 4.60 (apparent t $, 1 \mathrm{H}, J=3.4 \mathrm{~Hz}, \mathrm{CHOSi}$ ); 2.83 (dd, $1 \mathrm{H}, J=8.8$, 3.4, $\mathrm{Hz} \mathrm{C}=\mathrm{CCH}) ; 2.22$ (s, 3H, OC $\left.(\mathrm{O}) \mathrm{CH}_{3}\right) ; 2.13$ (m, 1H, CHCHOAc); 1.85-1.71 (m, 2H, CH$\left.{ }_{2} \mathrm{CHCH}_{3}, \mathrm{CHCH}_{3}\right) ; 1.20\left(\mathrm{~m}, 1 \mathrm{H}, \mathrm{CH}_{2} \mathrm{CHCH}_{3}\right) ; 1.02$ (s, 12H, $\left.\mathrm{SiC}\left(\mathrm{CH}_{3}\right)_{3}, \mathrm{CHCH}_{3}\right) ;{ }^{13} \mathrm{C}$ NMR (75 $\left.\mathrm{MHz}, \mathrm{CDCl}_{3}\right) \square$ 190.0, 169.7, 162.7, 136.4, 136.3, 136.2, 136.1, 134.2, 133.2, 129.9, 129.8, 127.8, 127.7, 127.6, 119.1, 96.3, 76.1, 44.7, 43.5, 40.2, 34.2, 27.3, 27.2, 21.7, 21.2, 19.6; HRMS (FAB+) exact mass calculated for $[\mathrm{M}+\mathrm{H}]^{+}\left(\mathrm{C}_{28} \mathrm{H}_{35} \mathrm{O}_{5} \mathrm{Si}\right)$ requires $\mathrm{m} / \mathrm{z} 479.2254$, found $\mathrm{m} / \mathrm{z}$ 479.2266. $[\square]_{D}^{25}=-13.82\left(\mathrm{c}=1.0, \mathrm{CHCl}_{3}\right)$.

\section{$(1 S, 4 a S, 5 S, 7 S, 7 \mathrm{a} R)$-1-acetoxy-1,4a,5,6,7,7a-hexahydro-5-(tert-butyl-diphenyl-} silanyloxy)-7-methylcyclopenta[c]pyran-4-carboxylic acid. Sodium hypochlorite (1.06 g, 9.4 mmol) was added to a stirred solution of $\mathrm{NaH}_{2} \mathrm{PO}_{4}(864 \mathrm{mg}, 6.3 \mathrm{mmol})$ in $\mathrm{H}_{2} \mathrm{O}(3.2 \mathrm{~mL})$. The resulting solution was added dropwise over $30 \mathrm{~min}$ to a stirred mixture of $(1 \mathrm{~S}, 4 \mathrm{a} S, 5 \mathrm{~S}, 7 \mathrm{~S}, 7 \mathrm{a} R)-4$ formyl-1,4a,5,6,7,7a-hexahydro-5-(tert-butyl-diphenyl-silanyloxy)-7-methylcyclopenta[c]pyran1-yl acetate $(300 \mathrm{mg}, 0.63 \mathrm{mmol})$ in 2-methyl butene $(4.2 \mathrm{~mL})$ and $\mathrm{tBuOH}(6.3 \mathrm{~mL})$. The resulting solution was stirred for $24 \mathrm{~h}$, at which time an additional portion of sodium hypochlorite (354 mg, $3.1 \mathrm{mmol})$ and $\mathrm{NaH}_{2} \mathrm{PO}_{4}(288 \mathrm{mg}, 2.1 \mathrm{mmol})$ in $\mathrm{H}_{2} \mathrm{O}(1.0 \mathrm{~mL})$ was added. After a further $24 \mathrm{~h}$ of stirring, the reaction was diluted with $10 \mathrm{~mL} \mathrm{H}_{2} \mathrm{O}$, and extracted with $3 \times 20 \mathrm{~mL} \mathrm{CH} \mathrm{Cl}_{2}$ and $3 \times 20 \mathrm{~mL} \mathrm{Et} 2 \mathrm{O}$. The combined organics were dried over $\mathrm{Na}_{2} \mathrm{SO}_{4}$ and concentrated in vacuo. Flash chromatography (5:1-1:6 pentane:Et ${ }_{2} \mathrm{O}$ ) afforded the title compound as a clear, colorless oil in 93\% yield (288 mg, $0.58 \mathrm{mmol}$ ). IR (film) 3073, 2958, 2932, 2859, 1764, 1682, 1634, 1428, 1367, 1287, 1193, 1089, 960.2, 912.0, 737.0, $704.3 \mathrm{~cm}^{-1}$; ${ }^{1} \mathrm{H}$ NMR (300 MHz, $\left.\mathrm{CDCl}_{3}\right) \square 7.76$ (s, 1H, OCH=C); 7.69-7.29 (m, 10H, SiPhH); 6.40 (d, 1H, $J$ $=8.7 \mathrm{~Hz}, \mathrm{CHOAc}) ; 4.52(\mathrm{~m}, 1 \mathrm{H}, \mathrm{CHOSi}) ; 2.77(\mathrm{dd}, 1 \mathrm{H}, J=8.4,3.0 \mathrm{~Hz}, \mathrm{C}=\mathrm{CCH}) ; 2.22(\mathrm{~s}, 3 \mathrm{H}$, $\left.\mathrm{OCOCH}_{3}\right) ; 2.15$ (m, 1H, CHCHOAc); 1.86-1.73 (m, 2H, $\left.\mathbf{C H}_{2} \mathrm{CHCH}_{3}, \mathrm{CHCH}_{3}\right) ; 1.24(\mathrm{~m}, 1 \mathrm{H}$, $\left.\mathrm{CH}_{2} \mathrm{CHCH}_{3}\right) ; 1.04\left(\mathrm{~s}, 12 \mathrm{H}, \mathrm{SiC}\left(\mathrm{CH}_{3}\right)_{3}, \mathrm{CHCH}_{3}\right) ;{ }^{13} \mathrm{C} \mathrm{NMR}\left(75 \mathrm{MHz}, \mathrm{CDCl}_{3}\right) \square$ 172.9, 169.9, 156.2, 136.4, 136.3, 136.2, 136.1, 134.5, 133.1, 129.9, 129.8, 127.8, 127.7, 127.6, 105.7, 95.5, 76.4, 44.9, 43.1, 42.3, 34.2, 27.3, 27.2, 21.7, 21.2, 19.4; HRMS (FAB+) exact mass calculated for $[\mathrm{M}+\mathrm{H}]^{+}\left(\mathrm{C}_{28} \mathrm{H}_{35} \mathrm{O} \mathrm{S}_{6} \mathrm{Si}\right)$ requires $\mathrm{m} / \mathrm{z}$ 495.2203, found $\mathrm{m} / \mathrm{z}$ 495.2225. $[\square]_{D}^{25}=-13.82(\mathrm{c}=1.0$, $\mathrm{CHCl}_{3}$ ). 
Iridolactone (4). A solution of $\mathrm{HF} \bullet$ pyridine $(2 \mathrm{~mL}, 70 \% \mathrm{HF})$ was added to a stirred solution of $(1 S, 4 \mathrm{a} S, 5 S, 7 S, 7 \mathrm{a} R)$-1-acetoxy-1,4a,5,6,7,7a-hexahydro-5-(tert-butyl-diphenylsilanyloxy)-7-methylcyclopenta[c]pyran-4-carboxylic acid (240 mg, $0.48 \mathrm{mmol})$ in THF (4 mL). After $10 \mathrm{~h}$ the reaction was diluted with $50 \mathrm{~mL} \mathrm{Et}_{2} \mathrm{O}$, and washed with $20 \mathrm{~mL}$ of a saturated $\mathrm{NaHCO}_{3}$ solution (Caution: violent bubbling). The aqueous layer was thoroughly extracted with $3 \times 20 \mathrm{~mL} \mathrm{CH}_{2} \mathrm{Cl}_{2}$ and $\mathrm{Et}_{2} \mathrm{O}$. The combined organics were dried over $\mathrm{Na}_{2} \mathrm{SO}_{4}$ and concentrated in vacuo. The resultant residue was then immediately dissolved in $\mathrm{CH}_{2} \mathrm{Cl}_{2}(10 \mathrm{~mL})$, at which time 1,3-dicyclohexyl carbodiimide $(150 \mathrm{mg}, 0.73 \mathrm{mmol})$ was added in one portion. After $15 \mathrm{~min}$, the reaction was concentrated in vacuo. Flash chromatography (5:1-1:4 pentane: $\left.\mathrm{Et}_{2} \mathrm{O}\right)$ afforded the title compound as a clear, colorless oil in $82 \%$ yield (95 mg, $0.40 \mathrm{mmol}$ ). IR (film) 2954, 2931, 2854, 1756, 1661, 1237, 1216, 1170, 1012, 972.2, 872.0 $\mathrm{cm}^{-1} ;{ }^{1} \mathrm{H}$ NMR $\left(300 \mathrm{MHz}, \mathrm{CDCl}_{3}\right) \square$ $7.31(\mathrm{~d}, 1 \mathrm{H}, 2.7 \mathrm{~Hz}, \mathrm{OCH}=\mathrm{C}) ; 6.35$ (s, 1H, CHOAc); 5.06 (apparent t, 1H, J = $4.6 \mathrm{~Hz}$, $\mathrm{CHOC}(\mathrm{O})) ; 3.42(\mathrm{~m}, 1 \mathrm{H}, \mathrm{C}=\mathrm{CCH}) ; 2.20-2.10$ (m, 4H, OCOCH, $\left.\mathrm{CHCHOAc}_{3}\right) 1.98$ (m, 1H, $\left.\mathrm{CH}_{2} \mathrm{CHCH}_{3}\right) ; 1.66\left(\mathrm{~m}, 1 \mathrm{H}, \mathrm{CHCH}_{3}\right) ; 1.24\left(\mathrm{~m}, 1 \mathrm{H}, \mathrm{CH}_{2} \mathrm{CHCH}_{3}\right) ; 1.07$ (d, 3H, J = $6.3 \mathrm{~Hz}$, $\left.\mathrm{CHCH}_{3}\right) ;{ }^{13} \mathrm{C} \mathrm{NMR}\left(75 \mathrm{MHz}, \mathrm{CDCl}_{3}\right) \square 170.2,169.2,148.3,103.6,88.8,81.1,45.0,42.0,38.1$, 31.8, 20.9, 17.6; HRMS (EI+) exact mass calculated for $[\mathrm{M}]^{+}\left(\mathrm{C}_{12} \mathrm{H}_{14} \mathrm{O}_{5}\right)$ requires $\mathrm{m} / \mathrm{z}$ 238.0841, found $m / z$ 238.0838. $[\square]_{D}^{25}=-229.2\left(\mathrm{c}=1.0, \mathrm{CHCl}_{3}\right)$.

Brasoside Tetraacetate. 1- $O$-(Trimethylsilyl)-2,3,4,6-tetra- $O$-acetyl- - -D-glucopyranose (31.5 mg, $0.075 \mathrm{mmol}$, prepared according to the method of Allevi $\left.{ }^{4}\right)$ and 4 (7.0 $\left.\mathrm{mg}, 0.030 \mathrm{mmol}\right)$ were added as benzene solutions to a schlenk flask under argon. The benzene was then frozen and sublimed. The remaining solid was redissolved in $\mathrm{CH}_{3} \mathrm{CN}(0.15 \mathrm{~mL})$ and cooled to $-30{ }^{\circ} \mathrm{C}$. At this time TMSOTf $(2.3 \square \mathrm{L}, 0.012 \mathrm{mmol})$ was added dropwise as a $5 \%$ solution in $\mathrm{CH}_{3} \mathrm{CN}$. After stirring at $-30^{\circ} \mathrm{C}$ for $3 \mathrm{~d}$, the reaction was quenched with $1 \mathrm{~mL} \mathrm{pH} 7$ buffer and extracted with $3 \times 10 \mathrm{~mL} \mathrm{Et}_{2} \mathrm{O}$. The combined organics were dried over $\mathrm{Na}_{2} \mathrm{SO}_{4}$ and concentrated in vacuo. Flash chromatography (1:1-1:3 pentane: $\left.\mathrm{Et}_{2} \mathrm{O}\right)$ afforded the title compound as a clear, colorless oil in $86 \%$ yield $(13.6 \mathrm{mg}, 0.026 \mathrm{mmol})$. The spectral data $\left({ }^{1} \mathrm{H}\right.$ and $\left.{ }^{13} \mathrm{C}\right)$ were in full accord with those reported for the natural isolate, ${ }^{5,6}$ except for the IR and HRMS which have not been described: IR (film) 2955, 2920, 2858, 1756, 1660, 1367, 1219, 1216, 1038, 1013, 972.8, 862.2 $\mathrm{cm}^{-1}$; HRMS (EI+) exact mass calculated for $[\mathrm{M}+\mathrm{H}]^{+}\left(\mathrm{C}_{24} \mathrm{H}_{31} \mathrm{O}_{13}\right)$ requires $\mathrm{m} / z$ 527.1765, found $m / z$ 527.1764. $[\square]_{D}^{25}=-270.9\left(\mathrm{c}=1.0, \mathrm{CHCl}_{3}\right)$; lit: $[\square]_{D}=-229\left(\mathrm{c}=0.9, \mathrm{CHCl}_{3}\right){ }^{5}$ 
Brasoside (2). A solution of $\mathrm{MeOH}: \mathrm{Et}_{3} \mathrm{~N}: \mathrm{H}_{2} \mathrm{O}(0.08 \mathrm{~mL}, 8: 1: 1)$ was added slowly to a $-15^{\circ} \mathrm{C}$ solution of Brasoside tetraacetate $(5.0 \mathrm{mg}, 0.007 \mathrm{mmol})$ in $\mathrm{CH}_{2} \mathrm{Cl}_{2}(0.04 \mathrm{~mL})$. After $3 \mathrm{~h}$ the reaction was quenched with $0.5 \mathrm{~mL}$ pH 7 buffer, and extracted with $3 \times 5 \mathrm{~mL}$ EtOAc. The combined organics were dried over $\mathrm{Na}_{2} \mathrm{SO}_{4}$ and concentrated in vacuo. Flash chromatography (9:1 EtOAc:MeOH) afforded the title compound as a white powder in $90 \%$ yield $(2.3 \mathrm{mg}, 0.0063$ $\mathrm{mmol})$. The spectral data $\left({ }^{1} \mathrm{H}\right.$ and $\left.{ }^{13} \mathrm{C}\right)$ were in full accord with those already reported (Table 2), ${ }^{7}$ except for the IR and HRMS which have not been described: IR (film) 2960, 2917, 2849, 1749, 1654, 1261, 1090, 1019, $799.0 \mathrm{~cm}^{-1}$; HRMS (FAB+) exact mass calculated for $[\mathrm{M}+\mathrm{H}]^{+}$ $\left(\mathrm{C}_{16} \mathrm{H}_{23} \mathrm{O}_{9}\right)$ requires $m / z$ 359.1342, found $m / z$ 359.1332. $[\square]_{D}^{25}=-181.0(\mathrm{c}=0.41$, EtOH $)$; lit: $[\square]_{D}$ $=-170(\mathrm{c}=0.97, \mathrm{EtOH})^{5} ;[\square]_{D}=-283(\mathrm{c}=1.4, \mathrm{EtOH})^{6}$.

(E)-2-hydroxyethyl 3-(4-(benzyloxy)phenyl)acrylate. Oxalyl chloride (8 mL, 2M solution in $\mathrm{CH}_{2} \mathrm{Cl}_{2}$ ) was added to a stirred solution of $p$-benzyloxycinammic acid $(2.89 \mathrm{~g}, 11.3$ mmol, prepared according to the method of Doherty $\left.{ }^{8}\right)$ in $\mathrm{CH}_{2} \mathrm{Cl}_{2}(45 \mathrm{~mL})$ at $0{ }^{\circ} \mathrm{C}$. A few drops of DMF were then added, and the resulting solution was stirred at $0^{\circ} \mathrm{C}$ for $1 \mathrm{~h}$ and at $23{ }^{\circ} \mathrm{C}$ for a further $1 \mathrm{~h}$. At this stage the solution was added dropwise via cannula to a stirred mixture of ethylene glycol (12.6 mL, $226 \mathrm{mmol})$, triethylamine (4 mL, $28 \mathrm{mmol})$, and DMAP (100 mg, 0.82 $\mathrm{mmol})$ in $\mathrm{CH}_{2} \mathrm{Cl}_{2}(110 \mathrm{~mL})$ cooled to $0{ }^{\circ} \mathrm{C}$. The resulting solution was stirred for $12 \mathrm{~h}$, at which time the reaction was quenched with a saturated solution of $\mathrm{NaHCO}_{3}(200 \mathrm{~mL})$, and after extraction the organic layer was washed with brine, dried over $\mathrm{Na}_{2} \mathrm{SO}_{4}$ and concentrated in vасио. Flash chromatography (4:1 $\mathrm{Et}_{2} \mathrm{O}$ :pentane) afforded the title compound as a white solid in 93\% yield (3.14 g, 10.5 mmol). IR (film) 3420, 2956, 2932, 2859, 1707, 1636, 1602, 1511, 1254, 1172, 984.1, 825.5 cm ${ }^{-1}$; ${ }^{1} \mathrm{H}$ NMR $\left(300 \mathrm{MHz}, \mathrm{CDCl}_{3}\right) \square 7.68(\mathrm{~d}, 1 \mathrm{H}, J=16.2 \mathrm{~Hz}, \operatorname{ArCH}=\mathrm{CH})$; 7.50-7.35 (m, 7H, ArH); $6.98(\mathrm{~d}, 2 \mathrm{H}, J=9.0 \mathrm{~Hz}, \operatorname{ArH}) ; 6.34(\mathrm{~d}, 1 \mathrm{H}, J=16.2 \mathrm{~Hz}, \operatorname{ArCH}=\mathrm{CH})$; $5.10\left(\mathrm{~s}, 2 \mathrm{H}, \mathrm{CH}_{2} \mathrm{Ph}\right) ; 4.35\left(\mathrm{~m}, 2 \mathrm{H}, \mathrm{CO}_{2} \mathrm{CH}_{2} \mathrm{CH}_{2}\right) ; 3.90\left(\mathrm{dd}, 2 \mathrm{H}, J=9.0,5.1 \mathrm{~Hz}, \mathrm{CO}_{2} \mathrm{CH}_{2} \mathrm{CH}_{2}\right)$; ${ }^{13} \mathrm{C}$ NMR $\left(75 \mathrm{MHz}, \mathrm{CDCl}_{3}\right) \square 167.9,160.9,145.3,136.7,130.2,128.9,128.4,127.7,127.4$, 115.5, 115.4, 70.3, 66.4, 61.5; HRMS $(\mathrm{FAB}+)$ exact mass calculated for $[\mathrm{M}+\mathrm{H}]^{+}\left(\mathrm{C}_{18} \mathrm{H}_{19} \mathrm{O}_{4}\right)$ requires $m / z$ 299.1283, found $m / z, 299.1276$.

Enolsilane (13). Dess-Martin periodinane $(2.15 \mathrm{~g}, 5.1 \mathrm{mmol})$ was added to a stirred solution of (E)-2-hydroxyethyl 3-(4-(benzyloxy)phenyl)acrylate (1.21 g, $4.06 \mathrm{mmol})$ in $\mathrm{CH}_{2} \mathrm{Cl}_{2}$ 
$(20 \mathrm{~mL})$. After $10 \mathrm{~h}$ the reaction was concentrated and extracted with $3 \times 50 \mathrm{~mL} \mathrm{Et} \mathrm{t}_{2} \mathrm{O}$. The combined organics were concentrated in vacuo, providing $1.15 \mathrm{~g}$ (95\% yield, $5.8 \mathrm{mmol}$ ) of the corresponding aldehyde, which was immediately redissolved in $\mathrm{CH}_{3} \mathrm{CN}(5 \mathrm{~mL})$. The resulting solution was added dropwise to a premixed solution of triethylamine $(3.75 \mathrm{~mL}, 27 \mathrm{mmol})$ and chlorotrimethylsilane $(2.44 \mathrm{~mL}, 19.2 \mathrm{mmol})$ in $\mathrm{CH}_{3} \mathrm{CN}(5 \mathrm{~mL})$. This mixture was stirred for $2 \mathrm{~h}$, at which time the reaction was concentrated in vacuo. Flash chromatography ( $3: 1$ pentane: $\left.\mathrm{Et}_{2} \mathrm{O}\right)$ on Iatrobeads afforded the title compound as a white solid in $82 \%$ yield $(1.75 \mathrm{~g}, 4.8 \mathrm{mmol})$. IR (film) 3117, 3065, 3035, 2958, 2931, 2898, 2860, 1732, 1682, 1634, 1601, 1511, 1270, 1158, 1122, 985.2, $843.5 \mathrm{~cm}^{-1}$; ${ }^{1} \mathrm{H}$ NMR $\left(300 \mathrm{MHz}, \mathrm{CDCl}_{3}\right) \square 7.74(\mathrm{~d}, 1 \mathrm{H}, J=16.2 \mathrm{~Hz}, \mathrm{ArCH}=\mathrm{CH})$; 7.51-7.36 (m, 7H, ArH); $6.98(\mathrm{~d}, 2 \mathrm{H}, J=8.7 \mathrm{~Hz}, \operatorname{ArH}) ; 6.74(\mathrm{~d}, 1 \mathrm{H}, J=3.6 \mathrm{~Hz}$, CH=CHOTMS $) ; 6.39(\mathrm{~d}, 1 \mathrm{H}, J=16.2 \mathrm{~Hz}, \operatorname{ArCH}=\mathrm{CH}) ; 5.84(\mathrm{~d}, 1 \mathrm{H}, J=3.6 \mathrm{~Hz}$, $\mathrm{CH}=\mathrm{CHOTMS}) ; 5.10$ (s, 2H, $\left.\mathrm{CH}_{2} \mathrm{Ph}\right) ; 0.25$ (s, 9H, $\left.\mathrm{Si}\left(\mathrm{CH}_{3}\right)_{3}\right) ;{ }^{13} \mathrm{C}$ NMR $\left(75 \mathrm{MHz}, \mathrm{CDCl}_{3}\right)$ 164.3, 161.0, 146.1, 136.6, 130.2, 130.0, 128.9, 128.4, 127.7, 127.4, 121.2, 115.5, 114.7, 70.3, 0.2 ; HRMS (FAB+) exact mass calculated for $[\mathrm{M}+\mathrm{H}]^{+}\left(\mathrm{C}_{21} \mathrm{H}_{25} \mathrm{O}_{4} \mathrm{Si}\right)$ requires $\mathrm{m} / z$ 369.1522, found $m / z, 369.1517$.

\section{2- $\boldsymbol{O}$-Benzylcoumaroyl-4,6-bis- $\boldsymbol{O}$-benzyloxy-C-D-glucopyranose $\quad(14) . \quad(2 R, 3 R)-3-$} Hydroxy-2,3-bis-(benzylyloxy)-propionaldehyde (12) (125 mg, $0.42 \mathrm{mmol}$, prepared according to the method of MacMillan ${ }^{9}$ using D-proline) was added as a solution in $2.0 \mathrm{~mL}$ of toluene to a flame-dried schlenk flask charged with finely divided magnesium bromide diethyl etherate (322 $\mathrm{mg}, 1.25 \mathrm{mmol}$, freshly prepared from magnesium turnings and dibromoethane in $\mathrm{Et}_{2} \mathrm{O}$ ) and 2.0 $\mathrm{mL}$ of toluene cooled to $-20{ }^{\circ} \mathrm{C}$. After stirring for 30 minutes at $-20{ }^{\circ} \mathrm{C}, \mathbf{1 3}(230 \mathrm{mg}, 0.63$ mmol) was added as a solution in $0.5 \mathrm{~mL}$ toluene. The suspension was stirred at $-20{ }^{\circ} \mathrm{C}$ for 2 hours, then allowed to warm to $4{ }^{\circ} \mathrm{C}$ over the course of 4 hours. After stirring for an additional 24 hours at $4{ }^{\circ} \mathrm{C}$, the reaction was acidified by the addition of $50 \mathrm{~mL}$ saturated aqueous $\mathrm{NH}_{4} \mathrm{Cl}$ and extracted with $\mathrm{Et}_{2} \mathrm{O}(3 \times 50 \mathrm{~mL})$. The combined organics were washed with $50 \mathrm{~mL}$ brine, dried over anhydrous $\mathrm{Na}_{2} \mathrm{SO}_{4}$ and concentrated in vacuo. The residue was taken up in $5 \mathrm{~mL}$ of 7:2:1 THF:water:trifluoroacetic acid at $0{ }^{\circ} \mathrm{C}$ and stirred for 30 minutes before being quenched with $50 \mathrm{~mL} 10 \% \mathrm{NaHCO}_{3}$, extracted with $2 \times 100 \mathrm{~mL} \mathrm{Et}_{2} \mathrm{O}$, dried over anhydrous $\mathrm{Na}_{2} \mathrm{SO}_{4}$, filtered and concentrated in vacuo. Crude ${ }^{1} \mathrm{H}$ NMR analysis indicated an 10:1 mixture of glucose:mannose derived diastereomers. Flash chromatography (1:1-4:1 $\mathrm{Et}_{2} \mathrm{O}$ :pentane) afforded 
the title compound as a white solid (163 mg, $0.27 \mathrm{mmol}$ ) in $65 \%$ yield, 8:1 $\square: \square$. IR (film) 3425 , 3064, 3032, 2924, 2869, 1710, 1633, 1603, 1511, 1454, 1251, 1171, 1058, 910.58, 828.2, 743.8, $697.6 \mathrm{~cm}^{-1}$; ${ }^{1} \mathrm{H}$ NMR $\left(300 \mathrm{MHz}, \mathrm{CDCl}_{3}\right) \square$-isomer: $\square 7.72(\mathrm{~d}, 1 \mathrm{H}, J=15.9 \mathrm{~Hz}, \mathrm{ArCH}=\mathrm{CH})$; 7.47$7.20(\mathrm{~m}, 17 \mathrm{H}, \mathrm{ArH}) ; 6.94(\mathrm{~d}, 2 \mathrm{H}, J=8.7 \mathrm{~Hz}, \mathrm{ArH}) ; 6.37$ (d, 1H, $J=15.9 \mathrm{~Hz}, \mathrm{ArCH}=\mathrm{CH}) ; 5.48$ (m, 1H, H1); 5.06 (m, 2H, CH $\mathbf{H}_{2} \mathrm{Ph}$ ); 4.87 (dd, 1H, $\left.J=10.2,3.9 \mathrm{~Hz}, \mathrm{H} 2\right) ; 4.85$ (d, 1H, $J=11.4 \mathrm{~Hz}$, $\mathrm{CH}_{2} \mathrm{Ph}$ ); 4.64-4.50 (m, 3H, CH $\left.\mathbf{H}_{2} \mathrm{Ph}\right)$; 4.24 (m, 1H, H6); 4.09 (m, 1H, H6); 3.72-3.53 (m, 3H, H3, $\mathrm{H} 4, \mathrm{H} 5) ;{ }^{13} \mathrm{C}$ NMR $\left(75 \mathrm{MHz}, \mathrm{CDCl}_{3}\right.$ ) $\square$-isomer: $\square$ 167.4, 161.0, 146.0, 138.4, 138.0, 136.6, 130.2, 128.9, 128.8, 128.7, 128.4, 128.3, 128.2, 128.0, 127.7, 127.4, 115.4, 114.9, 90.7, 78.5, 75.0, 74.0, 73.7, 72.0, 70.3, 70.0, 68.9; HRMS (FAB+) exact mass calcd for $[\mathrm{M}+\mathrm{H}]^{+}\left(\mathrm{C}_{36} \mathrm{H}_{37} \mathrm{O}_{8}\right)$ requires $\mathrm{m} / \mathrm{z}$ 597.2488, found $\mathrm{m} / \mathrm{z} 597.2512 ;[\square]_{D}^{25}=30.59$ ( $\mathrm{c}=1.0, \mathrm{CHCl}_{3}, 8: 1 \square: \square$ mixture).

2-O-Benzylcoumaroyl-3,4,6-tris- $O$-benzyloxy- $\square$-D-glucopyranose. Benzyl bromide $(0.132 \mathrm{~mL}, 1.1 \mathrm{mmol})$ was added to a solution of freshly prepared $\mathrm{Ag}_{2} \mathrm{O}(255 \mathrm{mg}, 1.1 \mathrm{mmol})$ and $12(131 \mathrm{mg}, 0.22 \mathrm{mmol})$ in $2.3 \mathrm{~mL}$ of $\mathrm{CH}_{2} \mathrm{Cl}_{2}$ stirred in the dark. After stirring for $18 \mathrm{~h}$, the reaction was filtered through a pad of celite and concentrated in vacuo. The resulting residue was redissolved in $\mathrm{MeOH}(3 \mathrm{~mL})$, at which time ammonium formate $(208 \mathrm{mg}, 3.3 \mathrm{mmol})$ and $10 \% \mathrm{Pd}$ on alumina $(220 \mathrm{mg}$ ) were added. The suspension was stirred for 10 hours, then filtered and concentrated in vacuo. Flash chromatography (1:1-3:1 Et $\mathrm{Et}_{2} \mathrm{O}$ :pentane) afforded the title compound as a white solid (103 mg, $0.15 \mathrm{mmol}$ ) in 68\% yield, 12:1 $\square: \square$. IR (film) 3424, 3063, $3030,2892,2868,1713,1631,1602,1510,1453,1249,1172,1060,827.6,736.4,697.1 \mathrm{~cm}^{-1} ;{ }^{1} \mathrm{H}$ NMR (300 MHz, $\left.\mathrm{CDCl}_{3}\right) \square$-isomer: $\square 7.70(\mathrm{~d}, 1 \mathrm{H}, J=15.9 \mathrm{~Hz}, \mathrm{ArCH}=\mathrm{CH}) ; 7.48-7.18(\mathrm{~m}, 22 \mathrm{H}$, $\operatorname{ArH}) ; 6.98(\mathrm{~d}, 2 \mathrm{H}, J=8.7 \mathrm{~Hz}, \operatorname{ArH}) ; 6.33(\mathrm{~d}, 1 \mathrm{H}, J=15.9 \mathrm{~Hz}, \operatorname{ArCH}=\mathrm{CH}) ; 5.50(\mathrm{t}, 1 \mathrm{H}, J=3.6$

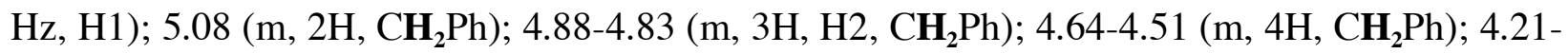
4.14 (m, 2H, H6); 3.86 (m, 1H, H3); 3.72-3.67 (m, 2H, H4, H5); $\left.{ }^{13} \mathrm{C} \mathrm{NMR} \mathrm{(75} \mathrm{MHz,} \mathrm{CDCl}\right) \square-$ isomer: $\square 166.8,161.0,145.7,138.7,138.3,138.0,136.7,130.2,128.9,128.8,128.7,128.6$, 128.4, 128.3, 128.2, 128.1, 128.0, 127.9, 127.8, 127.7, 127.5, 115.5, 115.2, 90.9, 80.2, 78.4, 77.8, 77.4, 76.9, 75.8, 75.4, 73.9, 73.7, 70.5, 70.3, 69.0; HRMS (EI+) exact mass calcd for $[\mathrm{M}]^{+}$ $\left(\mathrm{C}_{43} \mathrm{H}_{42} \mathrm{O}_{8}\right)$ requires $m / z$ 686.2880, found $m / z$ 686.2891; $[\square]_{D}^{25}=38.62\left(\mathrm{c}=1.0, \mathrm{CHCl}_{3}, 12: 1 \square: \square\right.$ mixture). 


\section{1-O-(Trimethylsilyl)-2-O-Benzylcoumaroyl-3,4,6-tris- $O$-benzyloxy-D-D-}

glucopyranose (15). Chlorotrimethylsilane $(27.8 \square \mathrm{L}, 0.22 \mathrm{mmol})$ was added dropwise over 20 $\min$ as a benzene $(1 \mathrm{~mL})$ solution to a refluxing mixture of triethylamine $(0.202 \mathrm{~mL}, 1.46 \mathrm{mmol})$ and 2-O-benzylcoumaroyl-3,4,6-tris- $O$-benzyloxy- $\square$-D-glucopyranose $(100 \mathrm{mg}, 0.146 \mathrm{mmol})$ in benzene $(2.9 \mathrm{~mL})$. After refluxing for $2 \mathrm{~h}$, the reaction was filtered through a pad of celite and concentrated in vacuo. Flash chromatography (3:1 pentane: $\left.\mathrm{Et}_{2} \mathrm{O}\right)$ on Iatrobeads afforded the title compound as a clear oil that solidifies to a white solid on standing in vacuo (101 mg, 0.133 mmol) in $91 \%$ yield. IR (film) 3064, 3032, 2958, 2868, 1716, 1634, 1603, 1511, 1454, 1251, 1150, 1068, 846.6, 736.6, $697.3 \mathrm{~cm}^{-1}$; ${ }^{1} \mathrm{H}$ NMR $\left(300 \mathrm{MHz}, \mathrm{CDCl}_{3}\right): \square 7.73(\mathrm{~d}, 1 \mathrm{H}, J=15.9 \mathrm{~Hz}$, $\operatorname{ArCH}=\mathrm{CH}) ; 7.55-7.25(\mathrm{~m}, 22 \mathrm{H}, \operatorname{ArH}) ; 7.03(\mathrm{~d}, 2 \mathrm{H}, J=8.7 \mathrm{~Hz}, \operatorname{ArH}) ; 6.33(\mathrm{~d}, 1 \mathrm{H}, J=15.9 \mathrm{~Hz}$, $\mathrm{ArCH}=\mathrm{CH}) ; 5.20-5.15\left(\mathrm{~m}, 3 \mathrm{H}, \mathrm{H1}, \mathrm{CH}_{2} \mathrm{Ph}\right) ; 4.92-4.61\left(\mathrm{~m}, 7 \mathrm{H}, \mathrm{H} 2, \mathrm{CH}_{2} \mathrm{Ph}\right) ; 3.85-3.76(\mathrm{~m}, 4 \mathrm{H}$, $\mathrm{H} 3, \mathrm{H} 5, \mathrm{H} 6) ; 3.62$ (m, 1H, H4); 0.24 (s, 9H, $\left.\left.\mathrm{Si}\left(\mathrm{CH}_{3}\right)_{3}\right) ;{ }^{13} \mathrm{C} \mathrm{NMR} \mathrm{(75} \mathrm{MHz,} \mathrm{CDCl}_{3}\right)$ : $\square$ 166.1, 160.9, 145.1, 138.6, 138.6, 138.4, 138.3, 136.8, 130.1, 129.0, 128.7, 128.6, 128.5, 128.4, 128.3, 128.2, 128.0, 127.9, 127.8, 127.7, 127.6, 115.8, 115.6, 96.3, 83.0, 78.4, 75.7, 75.4, 75.3, 75.2, 73.8, 70.4, 69.3, 0.48; HRMS (FAB+) exact mass calcd for $[\mathrm{M}-\mathrm{H}]^{+}\left(\mathrm{C}_{46} \mathrm{H}_{49} \mathrm{O}_{8} \mathrm{Si}\right)$ requires $\mathrm{m} / \mathrm{z}$ 757.3197, found $m / z$ 757.3174; $[\square]_{D}^{25}=44.67\left(\mathrm{c}=1.0, \mathrm{CHCl}_{3}\right)$.

2-O-Benzylcoumaroyl-3,4,6-tris- $O$-benzyloxy-brasoside (16). 15 (71 mg, $0.094 \mathrm{mmol}$ ) and 4 (15 mg, $0.063 \mathrm{mmol})$ were added as benzene solutions to a schlenk flask under argon. The benzene was then frozen and evaporated. The remaining solid was redissolved in $\mathrm{CH}_{3} \mathrm{CN}(0.25$ $\mathrm{mL})$ and cooled to $-30^{\circ} \mathrm{C}$. At this time TMSOTf $(6.1 \square \mathrm{L}, 0.031 \mathrm{mmol})$ was added dropwise as a $10 \%$ solution in $\mathrm{CH}_{3} \mathrm{CN}$. After $5 \mathrm{~d}$ stirring at $-30{ }^{\circ} \mathrm{C}$ the reaction was quenched with $2 \mathrm{~mL} \mathrm{pH} 7$ buffer, and extracted with $3 \times 15 \mathrm{~mL} \mathrm{Et}_{2} \mathrm{O}$. The combined organics were dried over $\mathrm{Na}_{2} \mathrm{SO}_{4}$ and concentrated in vacuo. Flash chromatography (50:1-25:1 $\left.\quad \mathrm{CH}_{2} \mathrm{Cl}_{2}: \mathrm{Et}_{2} \mathrm{O}\right)$ afforded the title compound as a clear, colorless oil in 74\% yield (40 mg, $0.047 \mathrm{mmol}$ ). IR (film) 3069, 3032, 2962, 2873, 1756, 1716, 1660, 1603, 1511, 1455, 1258, 1081, 800.0, 737.4, $698.4 \mathrm{~cm}^{-1} ;{ }^{1} \mathrm{H}$ NMR (300 MHz, $\left.\mathrm{CDCl}_{3}\right)$ : $\square 7.56(\mathrm{~d}, 1 \mathrm{H}, J=15.9 \mathrm{~Hz}, \mathrm{ArCH}=\mathrm{CH}) ; 7.49-7.15(\mathrm{~m}, 23 \mathrm{H}, \mathrm{ArH}, \mathrm{OCH}=\mathrm{C})$; $6.98(\mathrm{~d}, 2 \mathrm{H}, J=8.7 \mathrm{~Hz}, \operatorname{ArH}) ; 6.14(\mathrm{~d}, 1 \mathrm{H}, J=15.9 \mathrm{~Hz}, \mathrm{ArCH}=\mathrm{CH}) ; 5.52(\mathrm{~d}, 1 \mathrm{H}, J=0.9 \mathrm{~Hz}$, OCHO-Glucose); 5.12-4.53 (m, 11H, H1, H2, $\mathrm{CH}_{2} \mathrm{Ph}, \mathrm{CHOC}(\mathrm{O})$ ); 3.81-3.72 (m, 4H, H3, H5, H6); $3.58(\mathrm{~m}, 1 \mathrm{H}, \mathrm{H} 4), 3.42(\mathrm{dt}, 1 \mathrm{H}, J=6.9,2.7 \mathrm{~Hz}, \mathrm{OC}=\mathrm{CCH}) ; 2.05\left(\mathrm{~m}, 2 \mathrm{H}, \mathrm{CH}_{2} \mathrm{CHCH}_{3}\right.$, CHCHO-Glucose); $1.86\left(\mathrm{~m}, 1 \mathrm{H}, \mathrm{CHCH}_{3}\right) ; 1.54\left(\mathrm{~m}, 1 \mathrm{H}, \mathrm{CH}_{2} \mathrm{CHCH}_{3}\right) ; 1.00(\mathrm{~d}, 3 \mathrm{H}, J=6.3 \mathrm{~Hz}$, 
$\left.\mathrm{CHCH}_{3}\right) ;{ }^{13} \mathrm{C} \mathrm{NMR}\left(75 \mathrm{MHz}, \mathrm{CDCl}_{3}\right): \square 170.4,165.9,160.8,147.7,145.5,137.8,136.5,133.8$, $130.0,128.7,128.6,128.5,128.4,128.2$, 128.1, 128.0, 127.9, 127.8, 127.7, 127.6, 127.5, 127.1, 115.3, 114.8, 106.7, 104.0, 96.3, 92.9, 82.5, 81.0, 77.7, 77.2, 75.4, 75.2, 75.1, 73.5, 72.7, 70.1, 68.4, 45.1, 42.1, 38.1, 31.4, 17.8; HRMS (FAB+) exact mass calcd for $[\mathrm{M}+\mathrm{H}]^{+}\left(\mathrm{C}_{53} \mathrm{H}_{53} \mathrm{O}_{11}\right)$ requires $m / z 865.3588$, found $m / z 865.3563 ;[\square]_{D}^{25}=-37.42\left(\mathrm{c}=1.0, \mathrm{CHCl}_{3}\right)$.

Littoralisone (1). 16 (10 mg, $0.012 \mathrm{mmol})$ was dissolved in degassed benzene $(3.8 \mathrm{~mL})$ in a Pyrex flask under argon. This solution was exposed to $350 \mathrm{~nm}$ UV light (Hitachi UVA lamps, Luzchem 10 lamp photoreactor) with stirring for $2 \mathrm{~h}$. At this time the reaction was concentrated in vacuo, then redissolved in $\mathrm{EtOAc} / \mathrm{MeOH}(2: 1)$, and 10\% $\mathrm{Pd} / \mathrm{C}(5 \mathrm{mg})$ was added with stirring. This suspension was degassed and backfilled with $\mathrm{H}_{2}$ three times, at which point it was kept under a slight positive pressure of $\mathrm{H}_{2}$. After $30 \mathrm{~min}$, the reaction was filtered, and concentrated in vacuo. Flash chromatography (50:1-25:1 $\left.\mathrm{CH}_{2} \mathrm{Cl}_{2}: \mathrm{MeOH}\right)$ afforded the title compound as a white powder in $84 \%$ yield $(5.1 \mathrm{mg}, 0.010 \mathrm{mmol}) .{ }^{1} \mathrm{H}$ and ${ }^{13} \mathrm{C} \mathrm{NMR}$, IR and HRMS spectra confirm that synthetic and natural $\mathbf{1}$ are identical in all respects (Table 1). IR (film) $3391,1745,1635,1518,1448,1187,1076,972.4 \mathrm{~cm}^{-1}$. Synthetic $1[\square]_{D}^{25}=-46.1(c=0.4$, $\mathrm{MeOH})$, natural $1[\square]_{D}^{25}=-49.5(c=0.4, \mathrm{MeOH})^{10}$. HRMS $(\mathrm{FAB}+)$ exact mass calcd for $[\mathrm{M}+$ $\mathrm{H}]^{+}\left(\mathrm{C}_{25} \mathrm{H}_{29} \mathrm{O}_{11}\right)$ requires $m / z$ 505.1710, found $m / z$ 505.1699.

${ }^{1}$ Perrin, D. D.; Armarego, W. L. F. Purification of Laboratory Chemicals; $3^{\text {rd }}$ ed., Pergamon Press, Oxford, 1988.

${ }^{2}$ Pangborn, A. B.; Giardello, M. A,; Grubbs, R. H.; Rosen, R. K.; Timmers, F. J. Organometallics 1996, $15,1518$.

${ }^{3}$ Still, W. C.; Kahn, M.; Mitra, A. J. J. Org. Chem. 1978, 43, 2923.

${ }^{4}$ Allevi, P.; Anastasia, M.; Ciuffreda, P.; Bigatti, E.; MacDonald, P. J. Org. Chem. 1993, $58,4175$.

${ }^{5}$ Jensen, S. R.; Kirk, O.; Nielsen, B. J.; Norrestam, R. Phytochemistry 1987, 26, 1725.

${ }^{6}$ Schafer, B.; Rimpler, H. Z. Naturforsch 1979, 34, 311.

${ }^{7}$ Franke, A.; Rimpler, H. Phytochemistry 1987, 26, 3015.

${ }^{8}$ Doherty, D. G. J. Am. Chem. Soc. 1955, 77, 4887.

${ }^{9}$ Northrup, A. B.; Mangion, I. K.; Hettche, F.; MacMillan, D. W. C. Angew. Chem., Int. Ed. 2004, 43, 2152.

${ }^{10}$ Li, Y.-S.; Matsunaga, K.; Ishibashi, M.; Ohizumi, Y. J. Org. Chem. 2001, 66, 2165.

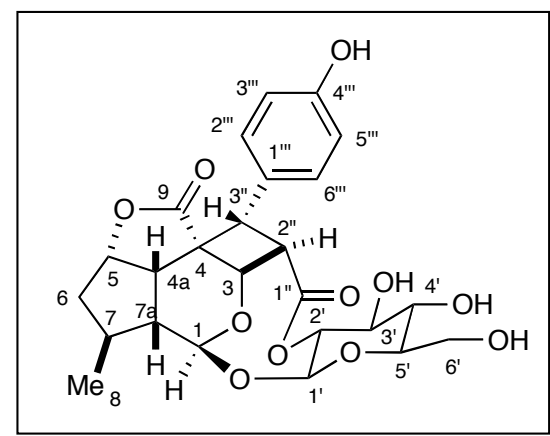


Table 1. ${ }^{1} \mathrm{H}$ and ${ }^{13} \mathrm{C}$ NMR Data for Natural ${ }^{10}$ and Synthetic $1^{\mathrm{a}}$

\begin{tabular}{|c|c|c|c|c|}
\hline \multirow[b]{2}{*}{ position } & \multicolumn{2}{|c|}{ Natural Littoralisone $\mathrm{b}^{\mathrm{b}}$} & \multicolumn{2}{|c|}{ Synthetic Littoralisone ${ }^{c}$} \\
\hline & ${ }^{13} \mathrm{C}(\square)$ & ${ }^{1} \mathrm{H}(\square), \mathrm{m}, \mathrm{J}(\mathrm{Hz})$ & ${ }^{13} \mathrm{C}(\square)$ & ${ }^{1} \mathrm{H}(\square), \mathrm{m}, \mathrm{J}(\mathrm{Hz})$ \\
\hline & & & & \\
\hline 1 & 96.45 & $5.23, \mathrm{~s}$ & 96.46 & $5.23, \mathrm{~s}$ \\
\hline 3 & 66.84 & $5.06, \mathrm{~d}, 11.1$ & 66.87 & $5.04, \mathrm{~d}, 10.8$ \\
\hline 4 & 48.13 & & 48.15 & \\
\hline $4 a$ & 43.87 & $3.35, \mathrm{dd}, 9.2,4.6$ & 43.90 & $3.33, \mathrm{dd}, 9.0,4.5$ \\
\hline 5 & 83.47 & $5.16, \mathrm{dd}, 5.0,4.6$ & 83.48 & $5.15, \mathrm{dd}, 6.8,4.4$ \\
\hline 6 & 43.23 & 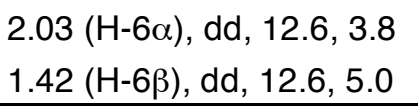 & 43.26 & $\begin{array}{l}2.02(\mathrm{H}-6 \square), \mathrm{dd}, 12.6,3.9 \\
1.41(\mathrm{H}-6 \square), \mathrm{dd}, 12.6,5.3\end{array}$ \\
\hline 7 & 33.21 & $1.49, \mathrm{~m}$ & 33.24 & $1.47, \mathrm{~m}$ \\
\hline $7 a$ & 46.98 & $1.70, \mathrm{dd}, 11.8,9.2$ & 47.01 & $1.70, \mathrm{dd}, 12.2,9.0$ \\
\hline 8 & 16.58 & $1.03, \mathrm{~d}, 5.7$ & 16.61 & $1.03, \mathrm{~d}, 5.7$ \\
\hline 9 & 176.94 & & 176.96 & \\
\hline $1^{\prime}$ & 100.37 & $4.83, d, 8.4$ & 100.39 & $4.81, d, 8.3$ \\
\hline $2^{\prime}$ & 80.49 & $4.73, \mathrm{dd}, 9.9,8.4$ & 80.51 & $4.72, \mathrm{dd}, 9.8,8.4$ \\
\hline 3 & 74.59 & $3.72, \mathrm{dd}, 9.9,8.4$ & 74.62 & $3.70, \mathrm{dd}, 9.8,8.4$ \\
\hline $4^{\prime}$ & 71.93 & $3.37, \mathrm{dd}, 9.9,8.4$ & 71.94 & $3.36, \mathrm{dd}, 9.8,8.4$ \\
\hline $5^{\prime}$ & 79.54 & $3.41, \mathrm{ddd}, 9.9,5.3,2.3$ & 79.57 & $3.40, \mathrm{ddd}, 9.8,5.3,2.0$ \\
\hline $6^{\prime}$ & 62.63 & $\begin{array}{l}3.68 \text { (H-6'a), dd, 11.8, } 5.3 \\
3.90 \text { (H-6’b), dd, 11.8, } 2.3\end{array}$ & 62.66 & $\begin{array}{l}3.67(\mathrm{H}-6 \text { 'a), dd, 12.2, } 5.3 \\
3.89\left(\mathrm{H}-6^{\prime} \mathrm{b}\right), \mathrm{dd}, 12.2,2.0\end{array}$ \\
\hline $1 "$ & 175.13 & & 175.14 & $0.97, d, 6.6$ \\
\hline 2" & 50.14 & $3.94, \mathrm{dd}, 11.1,4.6$ & 50.17 & $3.93, \mathrm{dd}, 11.0,4.6$ \\
\hline 3" & 49.65 & $4.15, d, 4.6$ & 49.66 & $4.14, d, 4.4$ \\
\hline $1, "$ & 128.37 & & 128.39 & \\
\hline $2 " ”$ & 130.32 & $7.06, \mathrm{dd}, 6.5,1.9$ & 130.35 & $7.05, \mathrm{dd}, 6.8,1.9$ \\
\hline 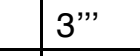 & 116.19 & $6.72, \mathrm{dd}, 6.5,1.9$ & 116.20 & $6.71, \mathrm{dd}, 6.8,1.9$ \\
\hline 4"” & 157.79 & & 157.84 & \\
\hline $5, "$ & 116.19 & $6.72, \mathrm{dd}, 6.5,1.9$ & 116.20 & $6.71, \mathrm{dd}, 6.8,1.9$ \\
\hline 6”"' & 130.32 & $7.06, \mathrm{dd}, 6.5,1.9$ & 130.35 & $7.05, \mathrm{dd}, 6.8,1.9$ \\
\hline
\end{tabular}

aSpectra were measured in $\mathrm{CD}_{3} \mathrm{OD}$. ${ }^{\mathrm{b} 1} \mathrm{H}$ NMR $(500 \mathrm{MHz}) ;{ }^{13} \mathrm{C}(125 \mathrm{MHz}) .{ }^{\mathrm{c} 1} \mathrm{H} \mathrm{NMR}(500 \mathrm{MHz})$; ${ }^{13} \mathrm{C}(125 \mathrm{MHz})$. 
${ }^{1} \mathrm{H}$ NMR of synthetic 1

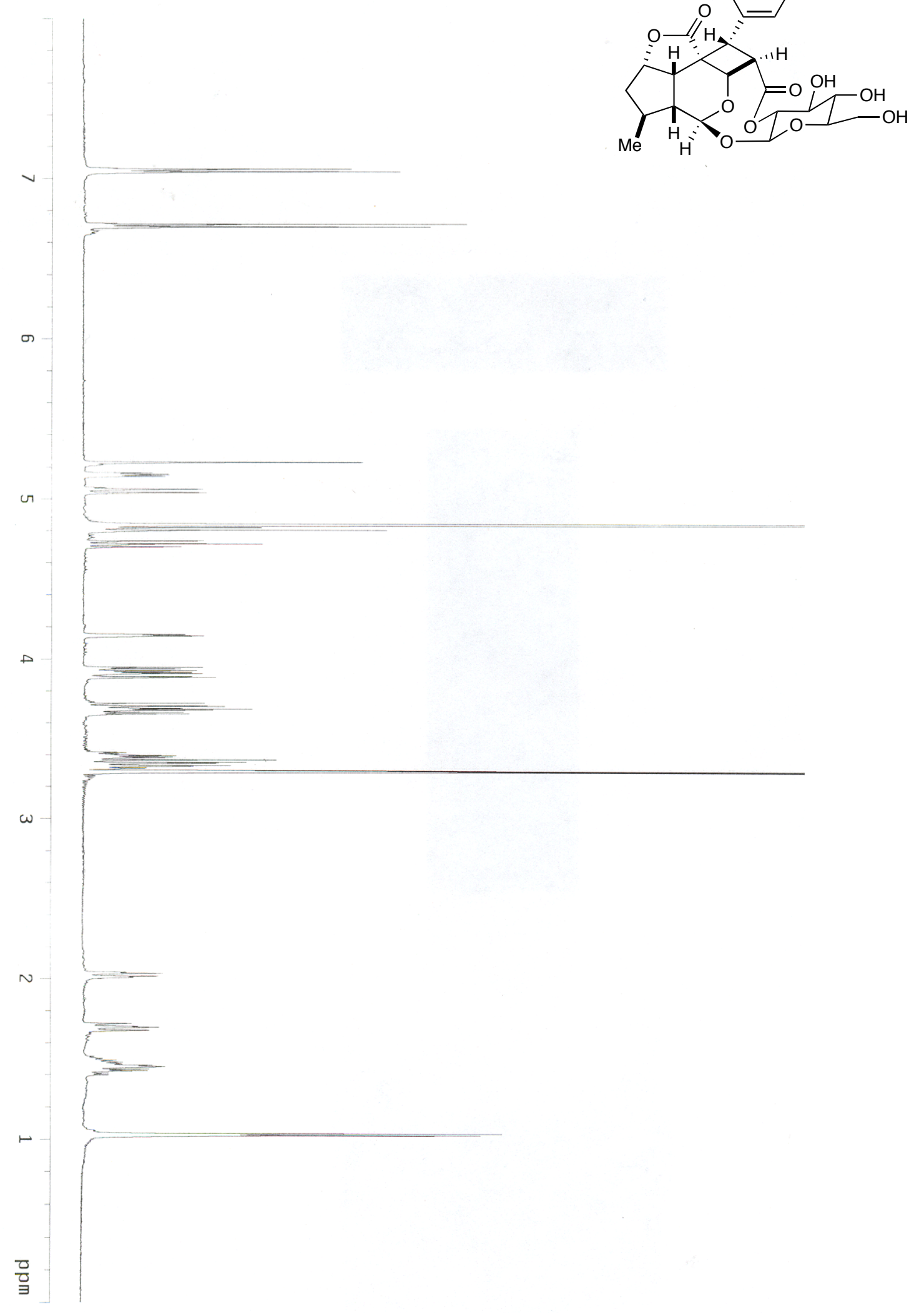


${ }^{13} \mathrm{C}$ NMR of synthetic 1
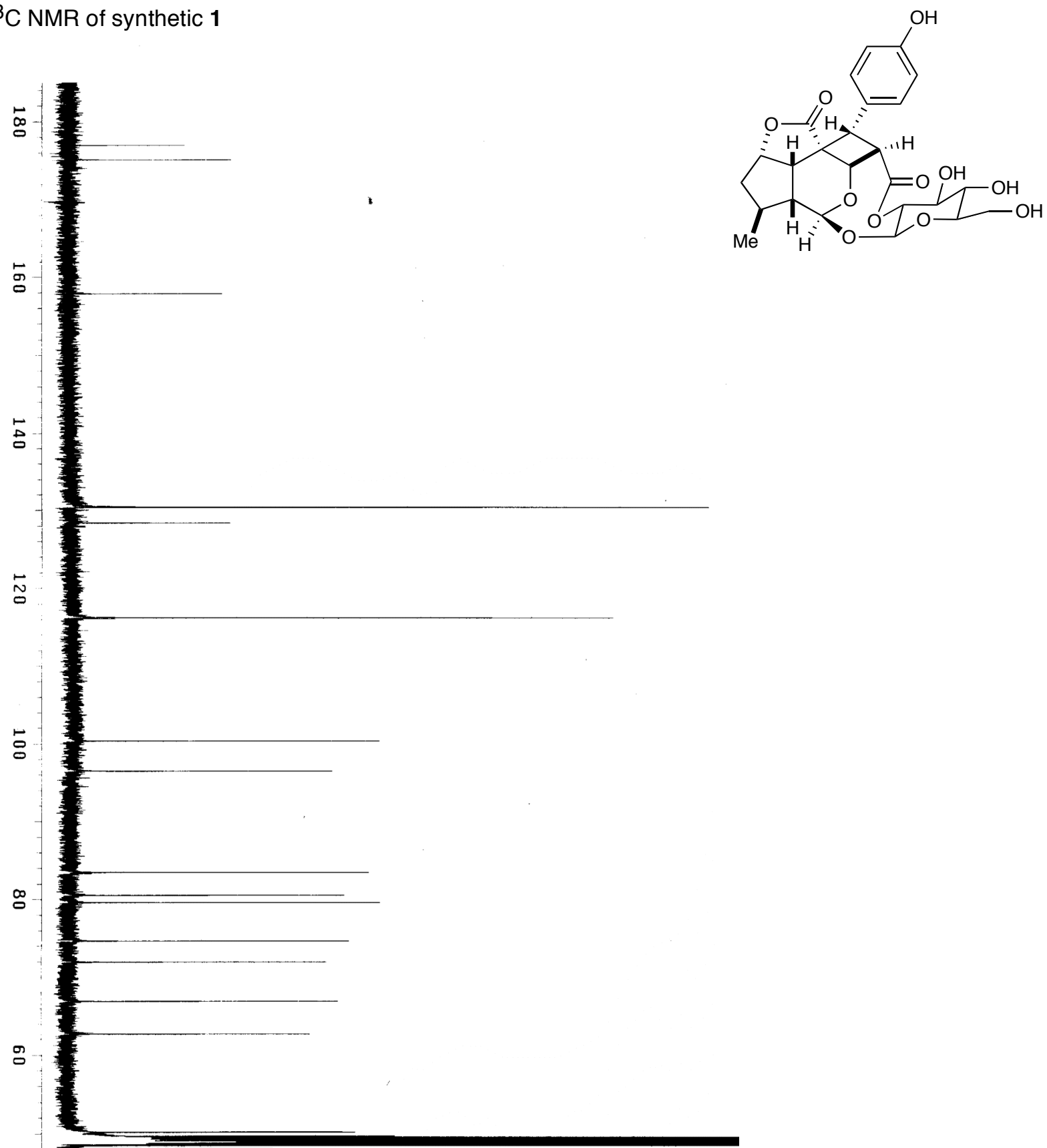


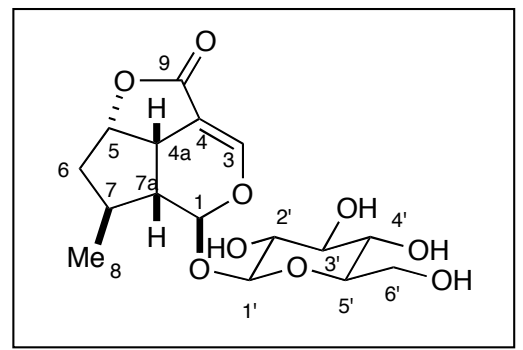

Table 1. ${ }^{1} \mathrm{H}$ and ${ }^{13} \mathrm{C}$ NMR Data for Natural ${ }^{5}$ and Synthetic $2^{\mathrm{a}}$

\begin{tabular}{|c|c|c|c|c|}
\hline \multirow[b]{2}{*}{ position } & \multicolumn{2}{|r|}{ Natural Brasoside ${ }^{b}$} & \multicolumn{2}{|r|}{ Synthetic Brasoside ${ }^{c}$} \\
\hline & $\begin{array}{l}{ }^{13} \mathrm{C} \\
(\square)\end{array}$ & ${ }^{1} \mathrm{H}(\square, \mathrm{m}, \mathrm{J}(\mathrm{Hz})$ & ${ }^{13} \mathrm{C}(\square)$ & ${ }^{1} \mathrm{H}(\square), \mathrm{m}, \mathrm{J}(\mathrm{Hz})$ \\
\hline & & & & \\
\hline 1 & 95.3 & $5.75, \mathrm{~s}$ & 95.5 & $5.75, \mathrm{~s}$ \\
\hline 3 & 150.7 & $7.45, \mathrm{~d}, 2.6$ & 150.8 & $7.44, d, 2.5$ \\
\hline 4 & 104.1 & & 104.2 & \\
\hline $4 a$ & 38.6 & $3.50, \mathrm{dt}, 7.2,2.5$ & 38.7 & $3.49, \mathrm{~m}$ \\
\hline 5 & 84.4 & $5.20, \mathrm{t}, 7.7$ & 84.6 & $5.20, t, 7.5$ \\
\hline 6 & 41.8 & $\begin{array}{c}2.08(\mathrm{H}-6 \square), \mathrm{dd}, 15.2,7.6 \\
1.73(\mathrm{H}-6 \square), \mathrm{ddd}, 15.1 \\
11.4,8.0\end{array}$ & 41.8 & 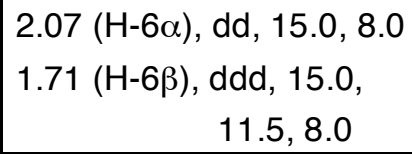 \\
\hline 7 & 32.3 & $1.94, \mathrm{~m}$ & 32.3 & $1.93, \mathrm{~m}$ \\
\hline $7 a$ & 45.5 & $2.18, \mathrm{ddd}, 11.0,6.8,0.8$ & 45.6 & $2.16, \mathrm{ddd}, 11.0,6.5,0.7$ \\
\hline 8 & 17.5 & $1.03, \mathrm{~d}, 7.0$ & 17.5 & $1.02, \mathrm{~d}, 6.5$ \\
\hline 9 & 175.0 & & 175.2 & \\
\hline $1^{\prime}$ & 99.4 & $4.91, d, 8.0$ & 99.5 & $4.90, \mathrm{~d}, 8.0$ \\
\hline 2' & 73.4 & -- & 73.4 & $3.30, \mathrm{dd}, 9.3,8.2$ \\
\hline 3' & 76.3 & -- & 76.3 & 3.55-3.47, m \\
\hline $4^{\prime}$ & 70.4 & -- & 70.4 & $3.41, \mathrm{dd}, 10.0,9.3$ \\
\hline 5 & 77.1 & -- & 77.2 & 3.55-3.47, m \\
\hline 6 & 61.5 & -- & 61.5 & $\begin{array}{l}3.74\left(\mathrm{H}^{\prime}{ }^{\prime} \mathrm{a}\right), \mathrm{dd}, 12.4,5.0 \\
3.94\left(\mathrm{H}-6^{\prime} \mathrm{b}\right), \mathrm{dd}, 12.4,2.2\end{array}$ \\
\hline
\end{tabular}

aSpectra were measured in $\mathrm{D}_{2} \mathrm{O} .{ }^{\mathrm{b} 1} \mathrm{H} \mathrm{NMR}(500 \mathrm{MHz}) ;{ }^{13} \mathrm{C}(125 \mathrm{MHz}) .{ }^{\mathrm{c} 1} \mathrm{H} \mathrm{NMR}(500 \mathrm{MHz})$; ${ }^{13} \mathrm{C}(125 \mathrm{MHz})$. 
${ }^{1} \mathrm{H}$ NMR of synthetic 2
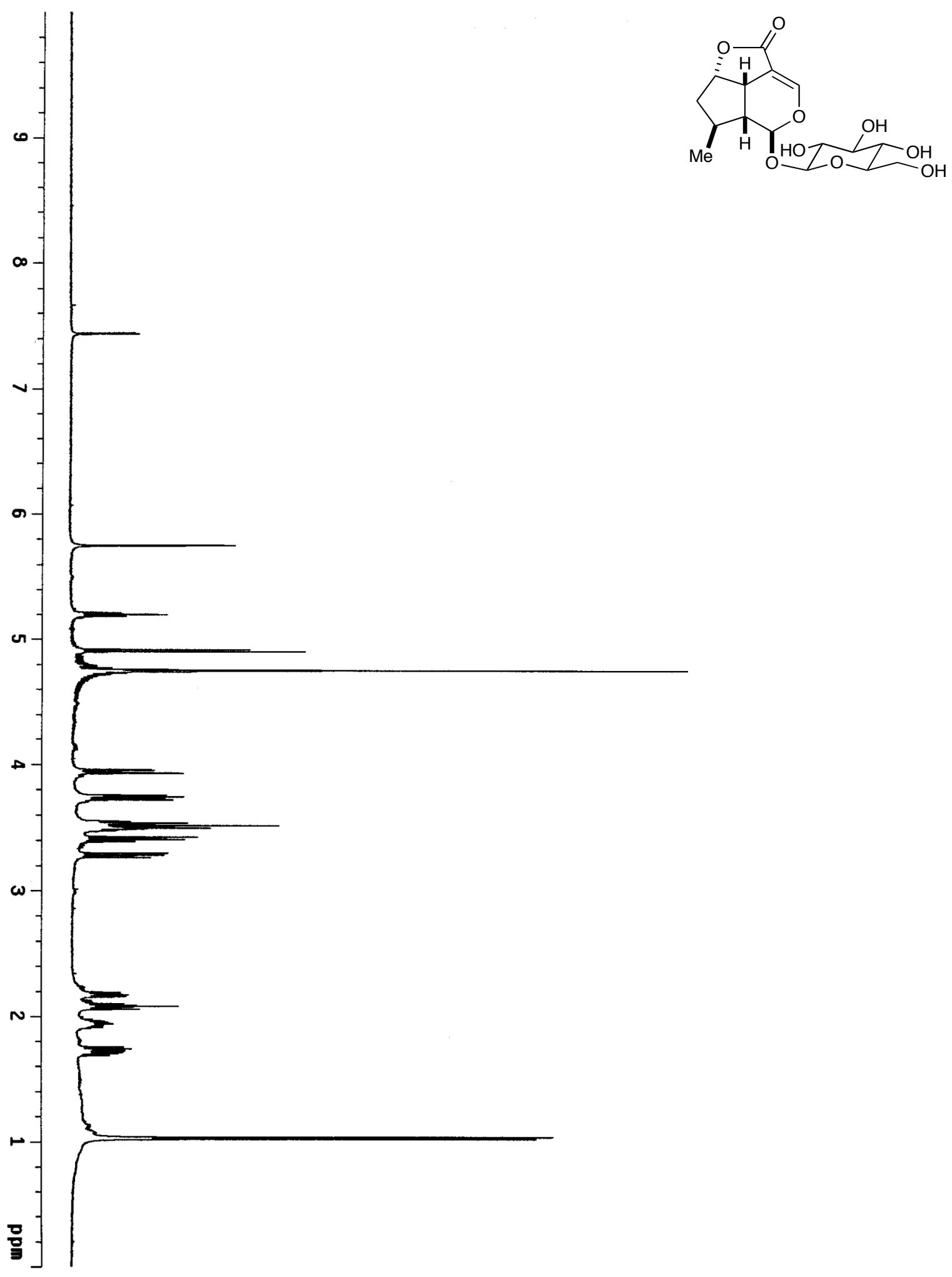
${ }^{13} \mathrm{C}$ NMR of synthetic 2
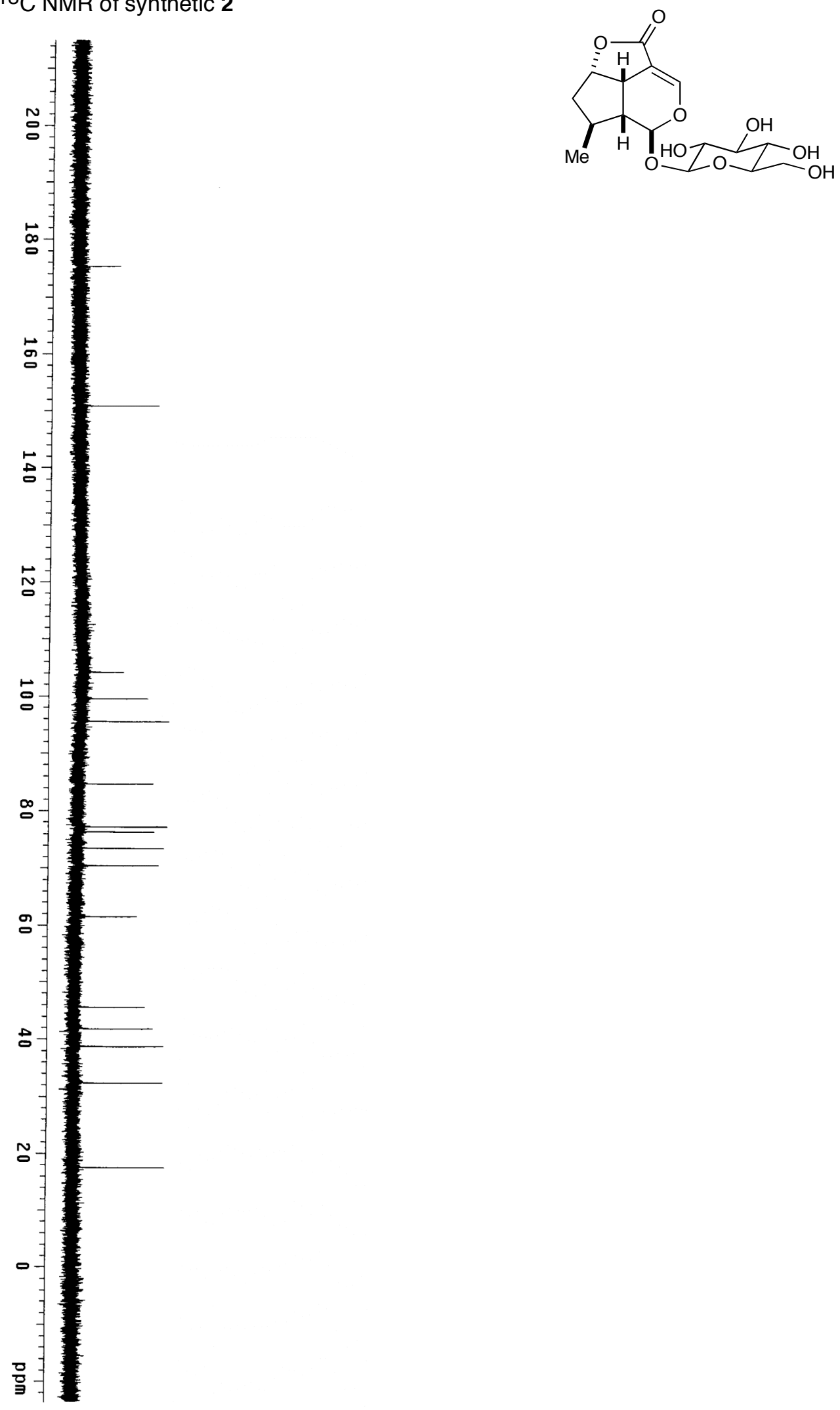